\title{
THE MINE DETECTION SYTEM COUPLED WITH A COMPACT TANDEM ACCELERATOR
}

\author{
J. H. Ha, Y. S. Cho, B. H. Choi, Y. K. Kim, J. H. Lee, Jae. S. Lee, KAERI, Taejon, 305-600, Korea
}

\section{Abstract}

We have been developing a mine detection system coupled with a $1 \mathrm{MV}$ compact tandem accelerator. This system will consist of a compact high current tandem accelerator, a target system, a detection system, a power generator system and a transportation system for application in the mine field. The tandem accelerator designed to be accelerated a proton of a $1.75 \mathrm{MeV}$ energy up to $10 \mathrm{~mA}$ beam current. We developed new mine detection method for a scattered $9.17 \mathrm{MeV}$ gamma-ray produced from the ${ }^{14} \mathrm{~N}(\gamma, \gamma){ }^{14} \mathrm{~N}$ photo-resonant reaction. The incident gamma-rays are produced by $1.75 \mathrm{MeV}$ proton beam bombarding at a ${ }^{13} \mathrm{C}$ target. New method can be separated a scattered gamma-rays from ${ }^{13} \mathrm{C}$ target gamma-rays within $0.1 \%$ energy resolution by using Doppler effect due to nucleus recoil mechanism.

\section{INTRODUCTION}

Several methods of mine and explosive detection were suggested, for example, NQR(Nuclear magnetic resonance), metal survey meter and so on. However, while applied to anti-personal mine which dose not contains any metal components, a conventional method based on metal survey is not effective. NQR method is deeply depended on the large electric quadrupole moment comparing with other stable element under ground, and this method was known that NQR detection frequency varied as a chemical composition and temperature. Our new mine and explosive detection technique is directly detected a nucleus by using the photo-nuclear resonant reaction for the ${ }^{14} \mathrm{~N}$. The main goal of this work is to confirm our new detection mechanism in principle by performing a basic nuclear resonant reaction experiment.

\section{NEW MINE/EXPLOSIVE DETECTION PRINCIPLE}

The mine/explosive detection mechanism is in two step.

0 Generation of $\gamma$-ray

$$
-p+{ }^{13} \mathrm{C}->14^{*}->14 \mathrm{~N}+\gamma
$$

0 Gamma resonance absorption and scattering

$$
-\gamma+{ }^{14} \mathrm{~N}->14^{*}{ }_{-}>14 \mathrm{~N}+\gamma \text { or }{ }^{13} \mathrm{C}+\mathrm{p}
$$

The photo-nuclear resonance gamma-rays are produced by nuclear reactions ${ }^{13} \mathrm{C}(\mathrm{p}, \gamma){ }^{14} \mathrm{~N}$, in which target is bombarded with a $1.75 \mathrm{MeV}$ proton beam extracted from the proton accelerator. To avoid other neighboring resonant gamma-rays, we selected a higher

*This work was supported by mid- and long-term project (Project No. 53564-00) of Ministry of Science and Technology in 2000. resonant energy of $9.17 \mathrm{MeV}$. The $9.17 \mathrm{MeV}$ gamma rays produced are absorbed or scattered when they react with ${ }^{14} \mathrm{~N}$ included in the mines and explosives. We can determine existence of mines or explosives by detecting the absorption and scattering gamma-ray signals.

In the study of explosives detection, we have surveyed the various present technologies and the requirements. The explosive detector based on the gamma resonance reaction should be relatively superior to the present ones in accuracy. The gamma resonance absorption in ${ }^{14} \mathrm{~N}$ rich compounds was verified successfully by using a Tandem accelerator at low beam current. But for the verification of the field application, a higher beam current over $10 \mathrm{~mA}$ is needed.

The major difficulty is that the energy of an absorbed and re-emitted gamma-ray from ${ }^{14} \mathrm{~N}(\gamma, \gamma){ }^{14} \mathrm{~N}$ reaction is almost same with that of a target gamma-ray come from ${ }^{13} \mathrm{C}(\mathrm{p}, \gamma){ }^{14} \mathrm{~N}$ resonant reaction. The over $30 \mathrm{~cm}$ thick $\mathrm{Pb}$ shield needed to overcome the ${ }^{13} \mathrm{C}$ target gamma-ray yield, and it is big burden for the system designer as a movable machine. The gamma-rays are produced by nuclear reactions that occur when an ${ }^{13} \mathrm{C}$ target is bombarded with a $1.75 \mathrm{MeV}$ proton beam, which is obtained from the proton accelerator. The $9.17 \mathrm{MeV}$ gamma-rays produced are absorbed or scattered when they react with ${ }^{14} \mathrm{~N}$ included in the mines and explosives. We can determine the position of mines or explosives by detecting the absorption and scattering of the gamma-rays.

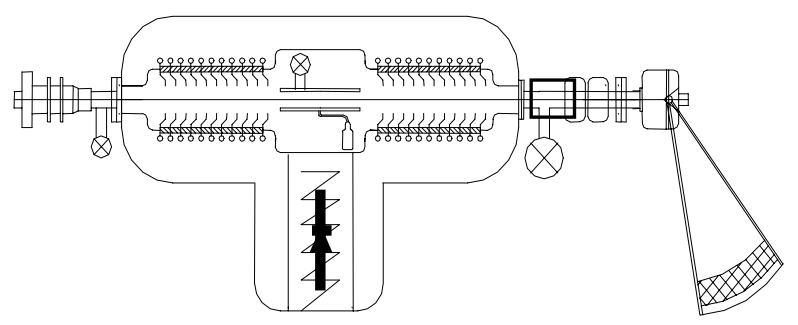

Fig. 1. Conceptual design of a mine/explosive detection system with Tandem accelerator.

\section{TANDEM ACCELERATOR DESIGN SPECIFIACTION}

Accelerator have to satisfied following specifications.

0 Particle : proton

o Beam Energy : $1.8 \mathrm{MeV} \pm 0.04 \mathrm{MeV}$

o Beam Current : $10 \mathrm{~mA} \pm 0.5 \mathrm{~mA}$

o Target diameter : $20 \mathrm{~mm}$

o Beam divergency : Less than 10mrad 


\section{PHYSICAL DATA FOR DETECTION ENVIROMENT}

Before performing the experiment, we have done an estimation calculation for our new detection principle. The properties of a $9.17 \mathrm{MeV}$ gamma-ray production ${ }^{13} \mathrm{C}$ target by $1.75 \mathrm{MeV}$ proton summarized as that natural abundance is $1.11 \%, \mathrm{~J}^{\pi}$ is $1 / 2^{-}$, density is $2.253 \mathrm{~g} / \mathrm{cm}^{3}$, $\mathrm{dE} / \mathrm{dx}=35.69 \mathrm{MeV} / \mathrm{mm}$, and range is $30.02 \mu \mathrm{m}$. The range and stopping power is calculated by TRIM code. The resonance width was reported as $122 \mathrm{eV}$ [1], and the calculated thick target yield as $0.64 \times 10^{-8}$ $[\gamma / \mathrm{p}]$. However, it does not consistent with each experimental data [1-5]. The $9.17 \mathrm{MeV}$ production yield as a unit of $[\gamma / \mathrm{p}]$ are varied with respect to several experiments, so in the simulation $0.88 \times 10^{-8}[\gamma / \mathrm{p}]$ is used, which was obtained by average some previous experiments, $0.74 \times 10^{-8}[2], 1.1 \times 10^{-8}[3], 1.01 \times 10^{-8}$ [4] and $0.63 \times 10^{-8}[5]$.

The underground condition was assumed as attenuation coefficient $0.0289 / \mathrm{cm}$, and explosive part of anti-personal mine dimension is that $1.5 \mathrm{~cm}$ or $5 \mathrm{~cm}$ radius and $1 \mathrm{~cm}$ or $5 \mathrm{~cm}$ height with a density of ${ }^{14} \mathrm{~N}$ element $0.5 \mathrm{~g} / \mathrm{cm}^{3}$

\section{DETECTION TEST EXPERIMENTS}

Experiments were performed several times by using KIGAM 1.7MV Tandem and SNU-AMS 3MV Tandetron accelerators. The main experiment was performed by using SNU-AMS Tadetron accelerator. Firstly, we reviewed previous experiments by using the $\mathrm{NaI}(\mathrm{Tl})$ with anti-cosmic ray by the thick plastic detectors. We used BGO scintillator to increase detection yield than $\mathrm{NaI}(\mathrm{Tl})$ scintillator. At least $30 \mathrm{~cm} \mathrm{~Pb}$ shield was required to distinguish the real signal of mine from observed signals..

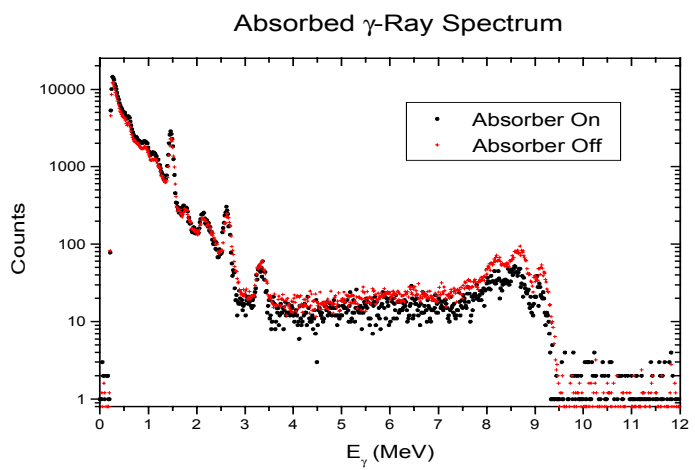

Fig.2. Resonant absorption reaction spectrum for the melamine mine/explosive emulator by $\mathrm{NaI}(\mathrm{Tl})$ scintillator..

The protons were accelerated to be $1.75 \mathrm{MeV}$, and the excitation function, angular distribution mine/explosive emulator like melamine or liquid nitrogen, photoabsorption and scattering were measured.

The proton beam current was over $1 \mu \mathrm{A}$, and bombarded on a enriched ${ }^{13} \mathrm{C}$ target foil $(99 \%)$ with 121.1 $\mu \mathrm{g} /$. thickness. The detectors were $2 " \times 2 "$ and $3 " \times 3 "$ $\mathrm{NaI}(\mathrm{Tl}), \mathrm{BGO}$, and 2" 2 " HP-Ge detector with BGO AntiCompton Shields. The target of $9.17 \mathrm{MeV}$ gamma-ray was $10 \mathrm{~cm}, 20 \mathrm{~cm}$ thick melamine or liquid nitrogen

The Doppler effect as a function of detection angle with respect to a proton beam is shown in Fig. 3. The solid line is the calculation result for the resonant reaction at $1.75 \mathrm{MeV}$ proton.

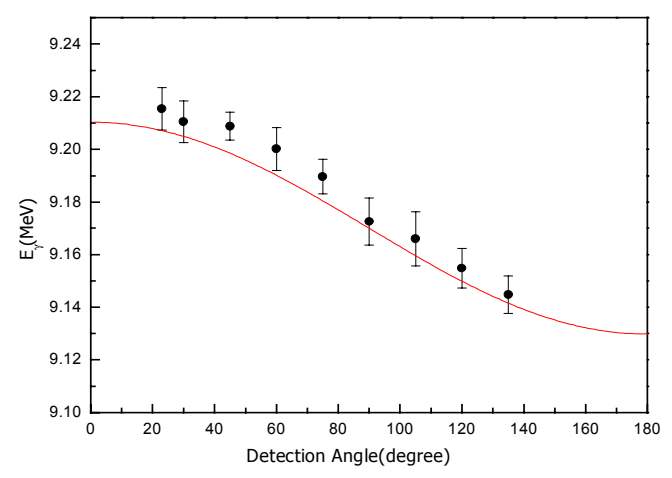

Fig. 3. Doppler shift effect due to nucleus recoil in resonant reaction.

We also developed new detection technique to detect the scattered gamma-ray from the nitrogen target by using the energy difference between the Doppler effected gamma-ray from the ${ }^{13} \mathrm{C}(\mathrm{p}, \gamma){ }^{14} \mathrm{~N}$ reaction, and the resonant absorbed and scattered gamma-ray from the ${ }^{14} \mathrm{~N}(\gamma, \gamma){ }^{14} \mathrm{~N}$ reaction, especially, $9.17 \mathrm{MeV}$ resonance state gamma-ray. In this experiment, the thickness of the enriched ${ }^{13} \mathrm{C}$ target was $121 \mu \mathrm{g} / \mathrm{cm}^{2}$ self-supporting foil, and we used liquid nitrogen with 20 liter as a resonant absorption target as mine/explosive emulator. We used a $\mathrm{HP}-\mathrm{Ge}$ detector with $30 \%$ efficiency and less $2 \mathrm{keV}$ energy resolution. Fig. 4. is the observed spectrum near single escape peak region.

\section{DISCUSSION}

The resonant gamma-ray production yield of the thick target with $121 \mu \mathrm{g} / \mathrm{cm}^{2}$ is maximized near $1.745 \mathrm{MeV}$, which is agreed well with previous result [1].

We will realize that the detection system based on the BGO detectors has a sufficient lead shield system. . Even though, the detection system based on the BGO scintillator has much more yield about 100 times than that on the HP-Ge detector, we want to prove our detection method and technique in principle by using Doppler effect, based on a HP-Ge detector guaranteed $2 \mathrm{keV}$ energy resolution at a several $\mathrm{MeV}$ energies. This system did not need any shield in principle, but when performing experiment $10 \mathrm{~cm} \mathrm{~Pb}$ brick was used between ${ }^{13} \mathrm{C}$ target and mine/explosive emulator. The major merit of this technique is that we can separated the gamma-ray from ${ }^{13} \mathrm{C}$ and mine/explosive emulator when we choose well detection angle of mine gamma-ray. This method is 


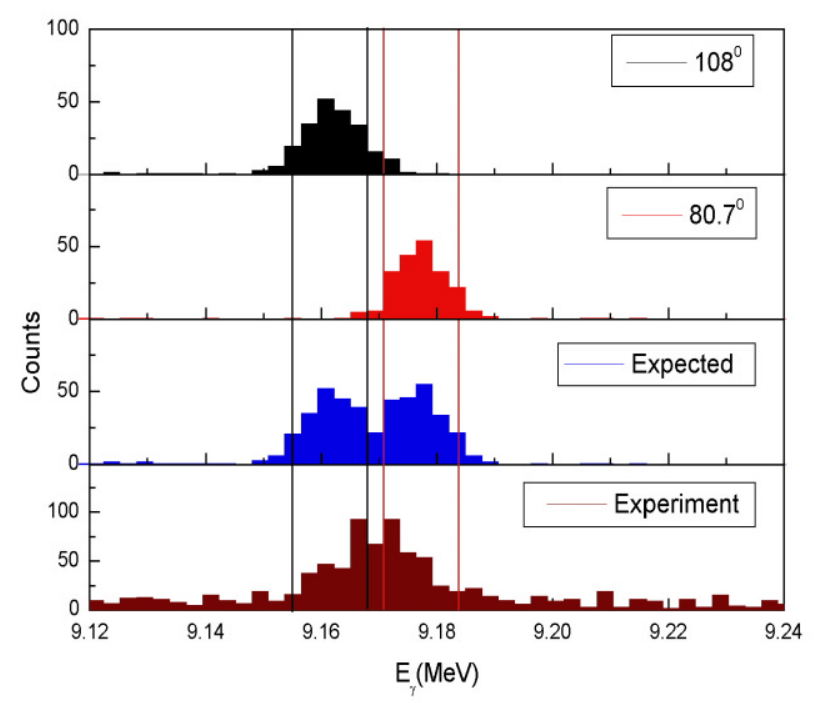

Fig. 4. The spectrum measured for the photo-nucleus resonant reaction. Spectrum at $108^{\circ}$ is measured at a off-resonanace angle and Spectrum $81^{\circ}$ is at an on-resonance angle.

deeply related on the Doppler effect, because the gammaray energies come from ${ }^{13} \mathrm{C}$ target are a function of detection angle, and the gamma-ray energy from mine emulator is almost a resonant energy. Such gamma-ray energy deviation is maximize when the detection angle is far from resonant angle.

The gamma-ray production and absorption process are the inverse mechanism, and the available angle of this mechanism is only $80.1^{\circ}$. The difference from Doppler effect between forward and backward detection angle is about $130 \mathrm{keV}$.

We also measured the angular distribution of the gamma-ray production by using the SNU-AMS tandetron accelerator. We used two HP-Ge detectors, in which one was used for the movable angular detector and the other was used for the normalization detector. Fortunately, the yield shows a maximum near at the detection angle $80^{\circ}$ near resonant angle.

The scattered gamma-ray yield from ${ }^{14} \mathrm{~N}(\gamma, \gamma){ }^{14} \mathrm{~N}$ resonant reaction is somewhat less than the expected yield calculated by using previous data. The measured scattered yield is $1.8 \mathrm{count} / \mathrm{sec} / 10 \mathrm{~mA}$ and the expected one is 4.2 count $/ \mathrm{sec} / 10 \mathrm{~mA}$. When we calculated the yield, some assumptions are used that thick target yield is $1 \times 10^{-8} \gamma$ $/ \mathrm{p}$, Hp-Ge detector absolute efficiency is $0.1 \%$ for the $59.2 \mathrm{~mm}($ dia $) \times 76.2 \mathrm{~mm}(\mathrm{~L})$ detection crystal dimension, the attenuation is $30 \%$ due to liquid nitrogen dewar case [6].

\section{CONCLUSION}

In this study of explosive detection, we have surveyed the various present technologies and the requirements. From the analysis, we have found that the explosive detector based on the gamma-ray resonance reaction should be relatively superior to the present ones in accuracy. The gamma resonance absorption in ${ }^{14} \mathrm{~N}$ rich compounds was verified successfully by using a Tandem accelerator. But for the verification of the field application, a higher beam current $(>10 \mathrm{~mA})$ is needed. The $9.17 \mathrm{MeV}$ gamma-ray absorbed about 12 times larger compared with the case without the nitrogen absorber.

Present work, we measured resonant energy and angular distribution, and photo-nucleus resonant gammarays by using $\mathrm{HP}-\mathrm{Ge}, \mathrm{BGO}$ and $\mathrm{NaI}(\mathrm{Tl})$ detectors.

We proved the new detection principle and observed scattered gamma-ray by using a new method based on energy difference originated from Doppler shift effect. We plan to measure the accurate experiment of the cross section and angular distribution by using KIGAM and SNU-AMS facilities.

We have plans for a new experiment at SNU-AMS facility, which is the largest heavy ion accelerator in Korea, to confirm and collect a meaningful physical nuclear data. The experimental setup will be used a detector shielding to suppress cosmic ray and target origin gamma-ray, and angular positioning system. The proton beam current will be larger than $10 \mu \mathrm{A}$. We will improve our data quality as a nuclear data point of view.

\section{REFERENCES}

[1] Table of Isotopes, 8th ed. (Wiley-Interscience City, 1996), Vol. I.

[2] S. S. Hanna and L. Meyer-Schutzmeister, Phys. Rev. 115(1959) 986

[3] J. D. Seagrave, Phys. Rev. 85 (1952) 197

[4] D. A. Close, NIM 113 (1973) 561

[5] LA-12777-MS, LANL Report(1994)

[6] Nuclear Reaction Analysis, J.B. Marion, F.C. Young(1967) 67 